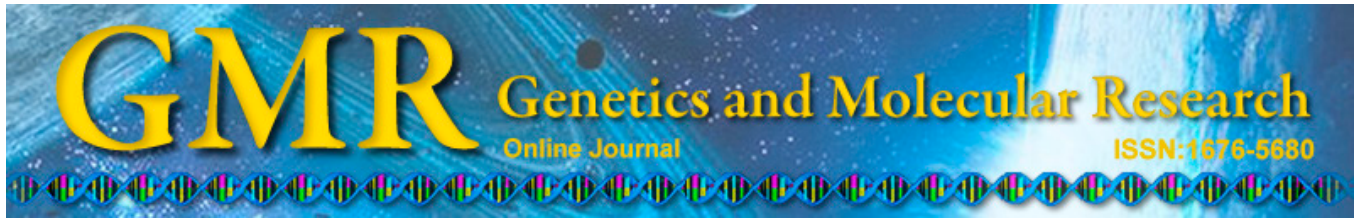

\title{
Cloning of an anthocyanidin synthase gene homolog from blackcurrant (Ribes nigrum L.) and its expression at different fruit stages
}

\author{
X.-G. Li ${ }^{1,2}$, J. Wang ${ }^{1,3}$ and Z.-Y. Yu ${ }^{2}$ \\ ${ }^{1}$ College of Forestry, Northeast Forestry University, Harbin, China \\ ${ }^{2}$ College of Horticulture, Northeast Agricultural University, Harbin, China \\ ${ }^{3}$ College of Food Sciences and Nutritional Engineering, \\ China Agricultural University, Beijing, China \\ Corresponding author: J. Wang \\ E-mail: junwang10944@163.com / jun_wang@cau.edu.cn
}

Genet. Mol. Res. 14 (1): 2726-2734 (2015)

Received September 26, 2014

Accepted February 20, 2015

Published March 31, 2015

DOI http://dx.doi.org/10.4238/2015.March.31.2

\begin{abstract}
Anthocyanidin synthase (ANS), a 2-oxoglutarate (2OG) and Fe(II)-dependent oxygenase, catalyzes the penultimate step in anthocyanin biosynthesis, from leucoanthocyanidins to anthocyanidins, the first colored compound in the anthocyanin pathway. In this study, a full-length, 1427-bp long cDNA named RnANS1, which is homologous to the anthocyanidin synthase gene, was cloned from blackcurrant using a homologous cloning strategy. RnANS1 is highly homologous to other plant ANS genes at both the nucleotide and amino acid sequence levels. The deduced protein contains domains conserved in the 2OG and Fe(II)-dependent oxygenase, and is phylogenetically closely related to Paeonia suffruticosa and Paeonia lactiflora. The expression of RnANS1 was upregulated during fruit maturation, and correlated with the accumulation of anthocyanins and soluble carbohydrates in the fruit. Further characterization of the structure and expression patterns of RnANS1 will clarify our understanding of anthocyanin biosynthesis in
\end{abstract}


blackcurrant, and support the development of molecular approaches to manipulate anthocyanin production in this plant.

Key words: Anthocyanin; Flavonoid; cDNA; Gene expression; Berry

\section{INTRODUCTION}

Anthocyanins, together with flavonols, flavones, and proanthocyanidins (PA), belong to the flavonoid class of secondary metabolites, which impart vivid colors to the flowers, fruits, and vegetative tissues of higher plants. Anthocyanins have well-characterized biomedicinal properties, including inhibition of cell proliferation, in addition to antimutagenic, antimicrobial, anti-inflammatory, and antihypertensive properties (Bohm, 1998; Harborne and Williams, 2000; de Pascual-Teresa and Sanchez-Ballesta, 2008). Anthocyanins and PAs are the two major functional flavonoids in plants, which counteract e ultraviolet radiation and microbial and fungal infections in the vegetative tissues of grapevines (Harborne and Williams, 2000; Chamkha et al., 2003), and contribute to the color, astringency, and quality of grape berries and wine (Chamkha et al., 2003; Bogs et al., 2005). Furthermore, their antioxidative activity has beneficial effects on human health, including protection against free radical-mediated injury and cardiovascular disease (Halliwell et al., 2005).

A key enzyme in the complex anthocyanin biosynthetic pathway is anthocyanidin synthase (ANS; EC1.14.11.19), which catalyzes the terminal oxidation of leucocyanidins to colored anthocyanins such as dihydroquercetins, using 2-oxoglutarate (2OG) as the substrate (Holton and Cornish, 1995; Xie et al., 2004). ANS is a member of the oxoglutarate irondependent dioxygenase family of enzymes, and is homologous to flavonol synthase (FLS) in the flavonoid biosynthetic pathway (Li et al., 2008). Homologs of ANS have been cloned and characterized in a variety of plants (Saito et al., 1999; Nakajima et al., 2001; Jin et al., 2005; Shimada et al., 2005; Wellmann et al., 2006). ANS contains a multicomponent active site containing metal, co-substrate, and two molecules of a substrate analog (dihydroquercetin), according to analysis of the crystal structure of the enzyme in Arabidopsis (Wilmouth et al., 2002). ANS expression has been shown to be regulated in specific tissues and cell types during plant development in response to a variety of stimuli, including stress (Gollop et al., 2001; Bogs et al., 2005; Castellarin et al., 2007; Deytieux et al., 2007).

Blackcurrant (Ribes nigrum L.) is a shrub in the family Grossulariaceae, which is commonly cultivated in temperate regions for its piquant black-colored berries, which at maturity are high in anthocyanins and vitamins. A better understanding of the molecular mechanisms underlying the regulation of anthocyanin in blackcurrant would help modify farming practices to improve fruit quality, as well as provide insights into the mechanisms involved in the anthocyanin metabolic pathway. Here, we report for the first time the cloning and expression analysis of the blackcurrant $A N S$ homolog RnANS1, in various stages of fruit development.

\section{MATERIAL AND METHODS}

\section{Materials}

Fruits and their peels were harvested from blackcurrant plants (cv. Broad) at five different developmental stages: prior to coloration, $20 \%$ colored, $50 \%$ colored, $80 \%$ colored, 
and mature (Figure 1), and grown at the germplasm nursery of the Horticultural Experiment Station at the Northeast Agricultural University (Harbin, China). Samples were flash-frozen in liquid nitrogen and stored at $-80^{\circ} \mathrm{C}$ for subsequent use.

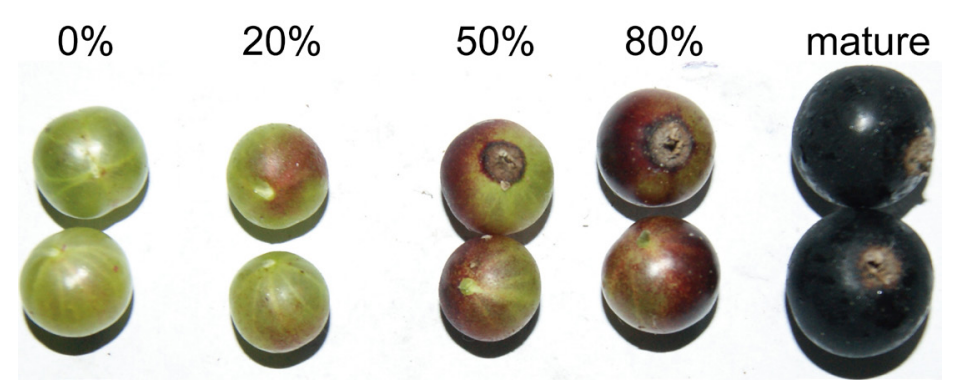

Figure 1. Blackcurrant berries at different stage of maturity (from left to right: green fruit, $0 \%$ veraison, $20 \%$ veraison, $50 \%$ veraison, $80 \%$ veraison, and mature).

\section{RNA extraction and cDNA synthesis}

Total RNA was extracted from frozen peel tissue using a modified CTAB method, and first-strand cDNAs were synthesized by reverse transcription using a PrimeScript ${ }^{\mathrm{TM}} \mathrm{RT}$ reagent kit (TaKaRa, Dalian, China), following the manufacturer protocol.

\section{Cloning of RnANS1}

A pair of degenerate primers was designed using sequences homologous to those of ANS homologs cloned from upland cotton (Gossypium hirsutum), cocoa (Theobroma cacao), and apple (Malus domestica). The sequence of the forward primer ANS-F was 5'-GC(T/A) $\mathrm{GC}(\mathrm{C} / \mathrm{T} / \mathrm{A})(\mathrm{A} / \mathrm{G}) \mathrm{TGGA}(\mathrm{T} / \mathrm{G}) \mathrm{TGGGG}(\mathrm{T} / \mathrm{A} / \mathrm{G}) \mathrm{GT}(\mathrm{G} / \mathrm{C}) \mathrm{ATG}-3$ ', and that of the reverse primer ANS-R was 5'-GCTTGAGTGGGAGGACTACTTCTTC-3'. The primers were used to amplify RnANS1 cDNA from reverse-transcribed cDNA in a $50-\mu \mathrm{L}$ reaction containing 1 $\mu \mathrm{L}$ Taq DNA polymerase ( $5 \mathrm{U} / \mu \mathrm{L}$; TaKaRa, Dalian, China), $5 \mu \mathrm{L}$ 10X Taq polymerase chain reaction (PCR) buffer, $4 \mu \mathrm{L}$ dNTP mixture ( $2.5 \mathrm{mM}$ each), $4 \mu \mathrm{L}$ cDNA template, and $2 \mu \mathrm{L}$ of each of the primers $(10 \mathrm{pmol})$. The amplification conditions were as follows: pre-denaturation at $95^{\circ} \mathrm{C}$ for $5 \mathrm{~min}$, followed by 35 cycles of denaturing at $95^{\circ} \mathrm{C}$ for $40 \mathrm{~s}$, annealing at $56^{\circ} \mathrm{C}$ for $30 \mathrm{~s}$, extension at $72^{\circ} \mathrm{C}$ for $1 \mathrm{~min}$, and a final extension at $72^{\circ} \mathrm{C}$ for $10 \mathrm{~min}$. The amplified products were separated by $1 \%$ agarose gel electrophoresis. Fragments were recovered from the gel, ligated into a pGEM $^{\circledR}$-T Easy Vector (Promega, Madison, WI, USA), and transformed into Escherichia coli TOP 10 cells (Promega). Positive clones were selected and sequenced at Sangon Biotech (Shanghai, China).

To obtain full-length RnANS1 cDNA, the 5' and 3' ends of the cDNA were amplified by RACE (rapid amplification of cDNA ends). Nested primers for the 3 ' and 5 ' ends were designed according to the conserved sequences obtained from the PCR products. They were 3RACEP1 (5'-GCAGTGGTATCAACGCAGAGTACTTTTTTTTTTTTTTTTTTTTTTTTTT-3'), 3RACEP2 (5'-GCAGTGGTATCAACGCAGAGTAC-3'), ANS3r1 (5'-CCAAATGTCCTCA ACCAGAACTAGCCCT-3'), ANS3r2 (5'-GAGTGCACTCACCTTCATACTCCAC-3'), 5RACEP1 (5'-GGCCACGCGTCGACTAGTACGGGGGGGGGGGGGG-3'), 5RACEP2 (5'-GGCCAC 
GCGTCGACTAGTAC-3'), ANS5r1 (5'-AACCCAAGCGATAAAGTTGAAAGCATGTC-3'), and ANS5r2 (5'-CTTGCGTACTCCGCTGTCACTTCAGTAT-3'). PCRs were carried under the same conditions as described above. The first and second rounds of nested PCR were run for 35 and 20 cycles, respectively. Amplified $3^{\prime}$ and 5' terminal fragments were recovered from the gels and sequenced as above. After removing vector sequences using Vector II (version 9.0), sequences were assembled using DNASTAR (Madison, WI, USA) to obtain full-length cDNA.

\section{Sequence analysis}

The open reading frame (ORF) of the ANS cDNA sequence was identified using ORF Finder (NCBI; http://www.ncbi.nlm.nih.gov/gorf/gorf.html). The molecular weight and isoelectric point of the deduced protein were determined using ProtParam (http://web.expasy.org/ protparam), and conserved regions were identified using BlastP (NCBI; http://www.ncbi.nlm. nih.gov/Structure/cdd/wrpsb.cgi). Protein family membership was predicted using Pfam 27.0 (http://pfam.sanger.ac.uk/), and multiple sequence alignment was carried out using Clustal X1.81. Phylogenetic tree analysis was carried out using MEGA (Tempe, Arizona, USA).

\section{Gene expression analysis}

Total RNA was extracted from blackcurrant berries at each developmental stage using the modified CTAB method. After DNase digestion to remove genomic DNA, the first strand of cDNA was reverse-transcribed using RNA as a template, as described above. Quantitative real-time PCR was performed using a MiniOpticon quantitative PCR instrument (BioRad, Hercules, CA, USA) to analyze the $A N S$ transcript levels, using $\mathrm{SYBR}^{\circledR}$ Green as the fluorescent dye. The primers used were ANS1-F (5'-GTGTCATGCACCTTGTCAACCATG-3') and ANS1-R (5'-CGTACTCCGCTGTCACTTCAGT-3'), which generated an expected 285bp long product. The Actin gene was amplified as an internal control using the primers actin-F (5'-CCGTCTCCAGAGTCCAGAACAATAC-3') and actin-R (5'-CTCACTGAAGCTCCTCT CAACCCAAAG-3'). Each set of samples included three duplicates. Levels of ANS transcripts were expressed relative to those of the Actin gene.

\section{Determination of anthocyanins and soluble carbohydrates}

Fruit anthocyanin content was determined as described previously (Pirie and Mullins, 1976). Briefly, $0.5 \mathrm{~g}$ fresh fruit peel was homogenized and extracted in $10 \mathrm{~mL} 1 \% \mathrm{HCl}$-methanol solution in the dark for $2 \mathrm{~h}$, after which absorbance of the extract was measured at 553 and $600 \mathrm{~nm}$. The difference between the absorbance values was used to calculate the relative anthocyanin content, where a single unit was calculated as $\left(\mathrm{OD}_{553}-\mathrm{OD}_{600}\right) \times 10$. The concentration of soluble carbohydrates was measured using an Abbe refractometer (2WAJ, Shanghai Optical Instrument Factory, Shanghai, China). All of the measurements were repeated three times.

\section{Statistical analyses}

All of the statistical analyses were performed using the SPSS 13.0 software (SPSS Inc., Chicago, IL, USA). Data are reported as means $\pm \mathrm{SD}$. 


\section{RESULTS}

\section{Cloning of the blackcurrant anthocyanidin synthase gene homolog}

The PCR amplification of reversely transcribed blackcurrant peel cDNA generated two products of approximately 344 and $50 \mathrm{bp}$ in length (Figure 2). Sequence analysis showed that the larger fragment was highly homologous to the sequences of anthocyanidin synthase genes in GenBank, but was not a full-length cDNA. 5' and 3' RACE-generated fragments of 638 and $617 \mathrm{bp}$ in length, respectively, were cloned, sequenced, and assembled into a full length cDNA of 1427 bp (GenBank accession No. KC493686), which was named RnANS1.

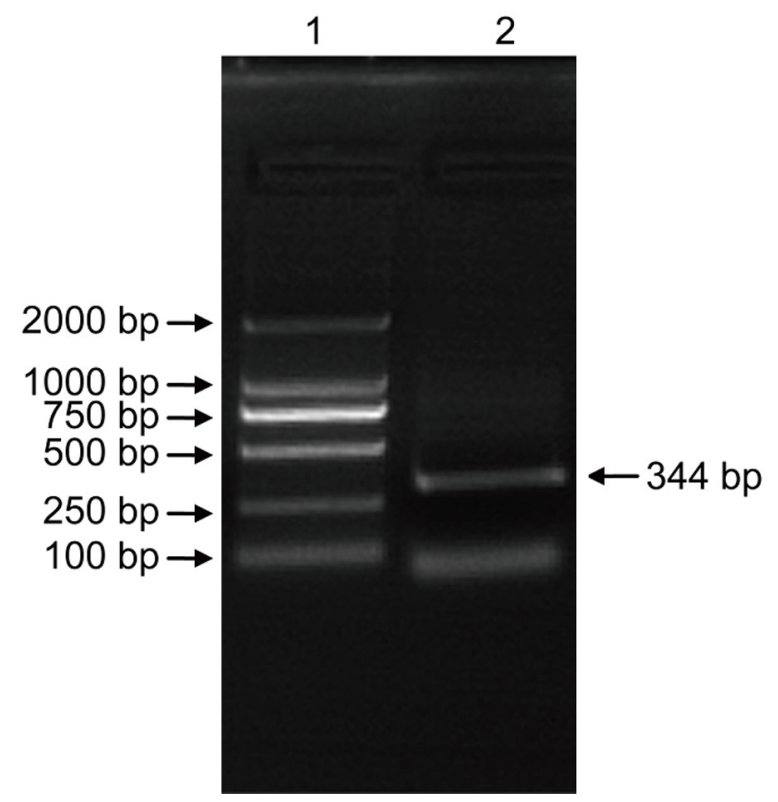

Figure 2. PCR result of $A N S$ gene homolog from blackcurrant. Lane $1=$ DNA molecular weight markers; lane $2=$ DNA amplified with ANS-specific degenerate primers.

\section{$R n A N S 1$ nucleotide and deduced amino acid sequence analysis}

The cDNA sequence of RnANS1 contained an ORF that was 1074 bp long, with an ATG located at nucleotides 103-105 and a termination codon (TGA) located at nucleotides 1174-1176. It had a 5'-untranslated region (UTR) that was 102-bp long (1-102 bp) and a 3'-UTR that was 251-bp long (1177-1427 bp). The ORF encoded a protein that contained 357 amino acids, with a predicted molecular weight of $40.51 \mathrm{kDa}$ and an isoelectric point of 5.45. To our knowledge, this is the first ANS homolog to be cloned from blackcurrant, hence its designation as RnANSI.

In silico protein domain analysis showed that the protein encoded by RnANS1 contained regions homologous to the DIOX_N and 2OG-FeII_Oxy DIOX_N domains (Figure 3). The former is highly conserved in the $\mathrm{N}$-terminal regions of proteins with $2 \mathrm{OG} / \mathrm{Fe}$ (II)-dependent dioxygenase activity, while the latter is conserved in members of the $2 \mathrm{OG}$ and Fe(II)-dependent 
oxygenase superfamily. Amino acid sequence alignment between RnANS1 and other ANS homologs showed that RnANS1 has high amino acid sequence identity with ANS in Paeonia suffruticosa (AEN71543.1, 83\%), P. lactiflora (AFI71900.1, 83\%), Ampelopsis grossedentata (AGO02175.1, 83\%), Vitis vinifera (ABV82967.1, 83\%), T. cacao (ADD51356.1, 82\%), Citrus sinensis (AAT02642.1, 80\%), and Pyrus communis (AGL50919.1, 77 \%) (Figure 4).

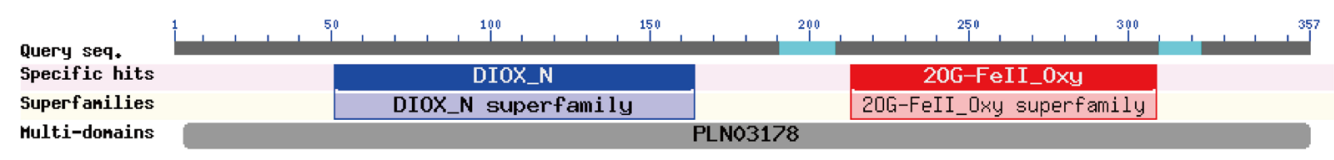

Figure 3. BLAST analysis of RnANS1 against GenBank sequences.

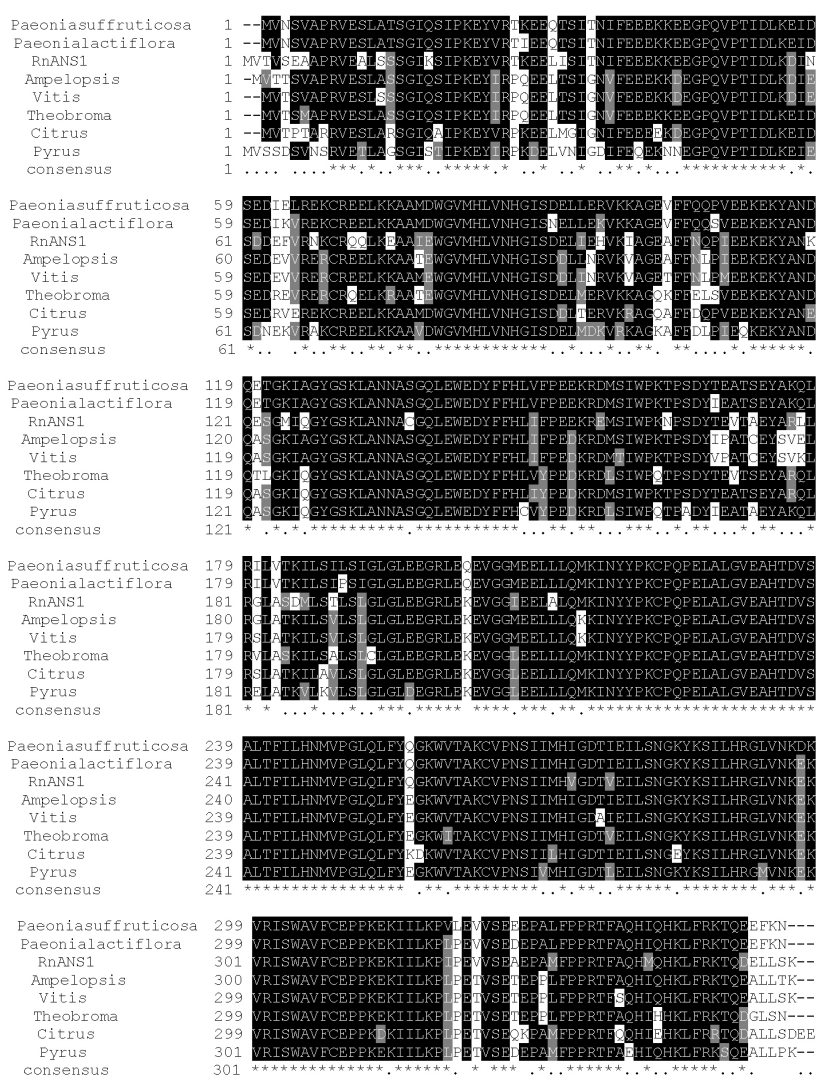

Figure 4. Amino acid sequence alignment of RnANS1 with ANS from other plant species. Stars indicate amino acids that are identical among the sequences compared. Nucleotides in black and gray shades are conservative across all or parts of the species examined, respectively. ${ }^{*}$ Consensus sequence.

Phylogenetic analysis indicated that RnANS1 is the most closely related to P. suffruticosa and P. lactiflora. It is also closely related to A. grossedentata (Ampelopsis), $V$. vinifera (grape), $T$. cacao (cocoa), and less closely related to C. sinensis (orange), and P. communis (pear) (Figure 5). 


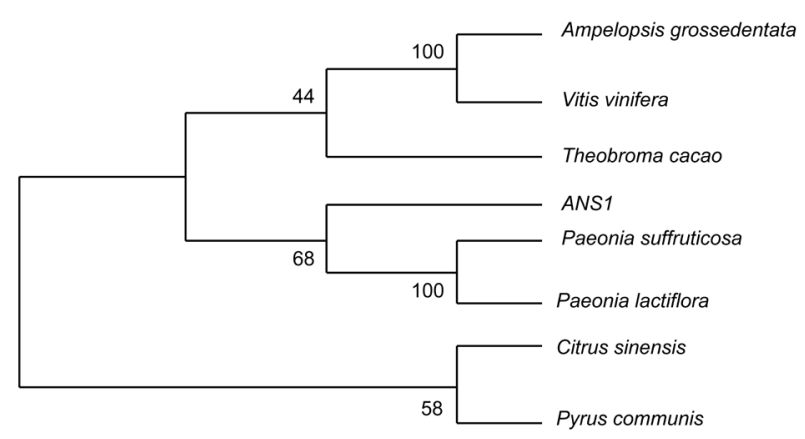

Figure 5. Phylogenetic analysis of ANS genes in blackcurrant and other plant species.

\section{Correlation between RnANS1 transcript levels and anthocyanin and soluble carbohydrate levels during fruit maturation}

Given its regulation of a key terminal reaction in flavonoid biosynthesis, we were interested in characterizing the regulation of RnANS1 expression in maturing blackcurrant fruit. RnANS1 transcript levels increased during each of the five developmental stages, particularly during the latter stage of fruit development (Figure 6a), reaching a peak when the fruit was fully mature (Figure 5). Fruit pigment and anthocyanin levels accumulated over the same period, beginning at the start of veraison, reaching a peak at $80 \%$ veraison, and decreasing slightly thereafter (Figure 6b), probably due to increased fruit weight. Similarly, fruit-soluble carbohydrate levels increased as the fruit matured (Figure 6c). RnANS1 expression during fruit maturation was positively correlated with both anthocyanin $(r=0.71)$ and soluble carbohydrate $(r=0.88)$ levels.

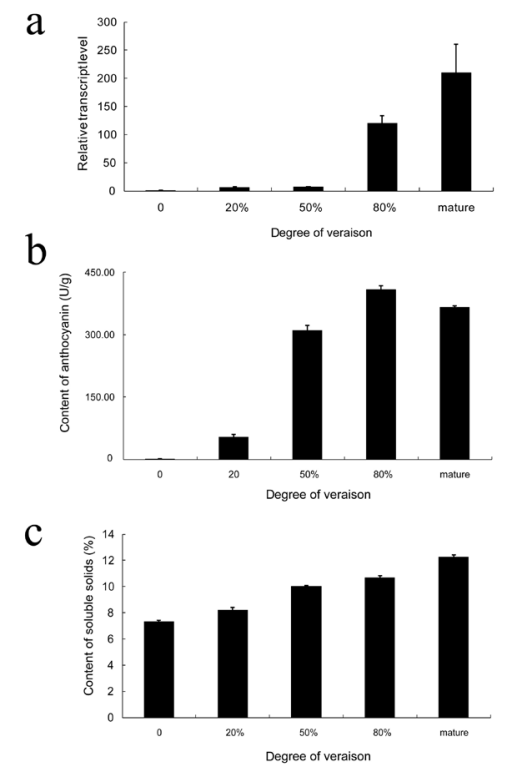

Figure 6. RnANS1 transcript levels (a), anthocyanin content (b), and soluble carbohydrate content (c) in the maturing blackcurrant fruit. Data are reported as means $\pm \mathrm{SD}$ (error bar). 


\section{DISCUSSION}

Using a homologous cloning strategy, we successfully amplified a full-length cDNA sequence that was highly identical to ANS gene sequences in GenBank, and encoded a key enzyme in anthocyanin biosynthesis with significant sequence similarity to three other flavonoid pathway enzymes (Turnbull et al., 2000, 2004). The sequence, which is to our knowledge the first reported in blackcurrant, was designated RnANS1, to reflect the fact that other currently uncharacterized ANS genes might be present in this plant. Consistent with its presence in a member of the Paeoniaceae family, phylogenetic analysis indicated that RnANS1 is the most closely related to $P$. suffruticosa and P. lactiflora.

The RnANS1 cDNA was deduced to encode a protein containing DIOX_N and 2OGFeII_Oxy DIOX_N domains, which are highly conserved in enzymes with 20G/Fe (II)-dependent dioxygenase activity, indicating that RnANS1 encodes an oxygenase that catalyzes the oxidation of leucocyanidins to colored anthocyanins. The involvement of this protein in anthocyanin synthesis is further supported by the fact that RnANS1 transcript levels and anthocyanin content increased in parallel during fruit development. These expression data indicate that the cloned gene is actively transcribed during the fruit development stages and developmentally regulated. These findings are consistent with results obtained in previous studies of other ANS genes (Boss et al., 1996; Deytieux et al., 2007).

It should be noted that this RnANS1 expression profile is in contrast to that in grape berries, in which $A N S$ transcript levels peak at an early stage of fruit development and decline gradually during the 30 to 60 days after full bloom (Wang et al., 2011), indicating that the expression profile of $A N S$ is species-specific. In addition to the correlation between RnANS1 transcript levels and anthocyanin content, we found that RnANS1 expression and soluble carbohydrate levels were also related, although the reason for this is unclear.

Given that the antioxidant activities of anthocyanins have beneficial implications for human health (Cheng et al., 2009; Singh et al., 2009), a better understanding of the synthesis of these molecules would facilitate their production for potential therapeutic and nutritional purposes. A previous study showed that transgenic silencing of ANS in apples largely ablated anthocyanin biosynthesis, resulting in a shift in the profiles of flavonoids and their related polyphenols, and significantly reduced plant viability (Szankowski et al., 2009). Consistent with this, the flavonoid profile that results from overexpression of $A N S$ has been shown to be associated with increased antioxidant potential in rice (Reddy et al., 2007). Our isolation of RnANS1 opens up the possibility of boosting blackcurrant anthocyanin content and flavonoid levels by genetic engineering, since the plant is now amenable to Agrobacterium-mediated transformation (Xu et al., 2009).

In summary, a full-length cDNA that was $1427 \mathrm{bp}$ in length, homologous to the anthocyanidin synthase gene, was cloned from blackcurrant. The deduced protein contained domains conserved in the $2 \mathrm{OG}$ and Fe(II)-dependent oxygenase, and was phylogenetically closely related to P. suffruticosa and P. lactiflora. RnANS1 expression increased during fruit maturation, and was correlated with the accumulation of anthocyanins and soluble carbohydrates in the fruit.

\section{ACKNOWLEDGMENTS}

Research supported by a grant (\#201103037) for special research funds for industrial technology and development for small berry crops in public welfare sectors (agriculture). 


\section{REFERENCES}

Bogs J, Downey MO, Harvey JS, Ashton AR, et al. (2005). Proanthocyanidin synthesis and expression of genes encoding leucoanthocyanidin reductase and anthocyanidin reductase in developing grape berries and grapevine leaves. Plant Physiol. 139: 652-663.

Bohm BA (1998). Introduction to Flavonoids. Harwood Academic Publishers, The Netherlands.

Boss PK, Davies C and Robinson SP (1996). Analysis of the expression of anthocyanin pathway genes in developing Vitis vinifera L. cv Shiraz grape berries and the implications for pathway regulation. Plant Physiol. 111: 1059-1066.

Castellarin SD, Pfeiffer A, Sivilotti P, Degan M, et al. (2007). Transcriptional regulation of anthocyanin biosynthesis in ripening fruits of grapevine under seasonal water deficit. Plant Cell Environ. 30: 1381-1399.

Chamkha M, Cathala B, Cheynier V and Douillard R (2003). Phenolic composition of champagnes from Chardonnay and Pinot Noir vintages. J. Agric. Food Chem. 51: 3179-3184.

Cheng S, Xu F and Wang Y (2009). Advances in the study of flavonoids in Ginkgo biloba leaves. J. Med. Plants Res. 3: $1248-1252$.

de Pascual-Teresa S and Sanchez-Ballesta MT (2008). Anthocyanins: from plant to health. Phytochem. Rev. 7: 281-299.

Deytieux C, Geny L, Lapaillerie D, Claverol S, et al. (2007). Proteome analysis of grape skins during ripening. J. Exp. Bot. 58: 1851-1862.

Gollop R, Farhi S and Perl A (2001). Regulation of the leucoanthocyanidin dioxygenase gene expression in Vitis vinifera. Plant Sci. 161: 579-588.

Halliwell B, Rafter J and Jenner A (2005). Health promotion by flavonoids, tocopherols, tocotrienols, and other phenols: direct or indirect effects? Antioxidant or not? Am. J. Clin. Nutr. 81: 268S-276S.

Harborne JB and Williams CA (2000). Advances in flavonoid research since 1992. Phytochemistry 55: 481-504.

Holton TA and Cornish EC (1995). Genetics and biochemistry of anthocyanin biosynthesis. Plant Cell 7: 1071-1083.

Jin Z, Grotewold E, Qu W, Fu G, et al. (2005). Cloning and characterization of a flavanone 3-hydroxylase gene from Saussurea medusa. DNA Seq. 16: 121-129.

Li Y, Zhao T and Chen S (2008). Anthocyanin biosynthesis and color regulation. Life Sci. 20: 147-152.

Nakajima J, Tanaka Y, Yamazaki M and Saito K (2001). Reaction mechanism from leucoanthocyanidin to anthocyanidin 3-glucoside, a key reaction for coloring in anthocyanin biosynthesis. J. Biol. Chem. 276: 25797-25803.

Pirie A and Mullins MG (1976). Changes in anthocyanin and phenolics content of grapevine leaf and fruit tissues treated with sucrose, nitrate, and abscisic acid. Plant Physiol. 58: 468-472.

Reddy AM, Reddy VS, Scheffler BE, Wienand U, et al. (2007). Novel transgenic rice overexpressing anthocyanidin synthase accumulates a mixture of flavonoids leading to an increased antioxidant potential. Metab. Eng. 9: 95-111.

Saito K, Kobayashi M, Gong Z, Tanaka Y, et al. (1999). Direct evidence for anthocyanidin synthase as a 2-oxoglutaratedependent oxygenase: molecular cloning and functional expression of cDNA from a red forma of Perilla frutescens. Plant J. 17: 181-189.

Shimada S, Inoue YT and Sakuta M. (2005). Anthocyanidin synthase in non-anthocyanin-producing Caryophyllales species. Plant J. 44: 950-959.

Singh K, Kumar S, Yadav SK and Ahuja PS (2009). Characterization of dihydroflavonol 4-reductase cDNA in tea [Camellia sinensis (L.) O. Kuntze]. Plant Biotechnol. Rep. 3: 95-101.

Szankowski I, Flachowsky H, Li H, Halbwirth H, et al. (2009). Shift in polyphenol profile and sublethal phenotype caused by silencing of anthocyanidin synthase in apple (Malus sp.). Planta 229: 681-692.

Turnbull JJ, Sobey WJ, Aplin RT, Hassan A, et al. (2000). Are anthocyanidins the immediate products of anthocyanidin synthase? Chem. Commun. 24: 2473-2474.

Turnbull JJ, Nakajima J, Welford RW, Yamazaki M, et al. (2004). Mechanistic studies on three 2-oxoglutarate-dependent oxygenases of flavonoid biosynthesis: anthocyanidin synthase, flavonol synthase, and flavanone $3 \beta$-hydroxylase. $J$. Biol. Chem. 279: 1206-1216.

Wang H, Wang W, Li H, Zhang P, et al. (2011). Expression and tissue and subcellular localization of anthocyanidin synthase (ANS) in grapevine. Protoplasma 248: 267-279.

Wellmann F, Griesser M, Schwab W, Martens S, et al. (2006). Anthocyanidin synthase from Gerbera hybrida catalyzes the conversion of (+)-catechin to cyanidin and a novel procyanidin. FEBS Lett. 580: 1642-1648.

Wilmouth RC, Turnbull JJ, Welford RW, Clifton IJ, et al. (2002). Structure and mechanism of anthocyanidin synthase from Arabidopsis thaliana. Structure 10: 93-103.

Xie DY, Jackson LA, Cooper JD, Ferreira D, et al. (2004). Molecular and biochemical analysis of two cDNA clones encoding dihydroflavonol-4-reductase from Medicago truncatula. Plant Physiol. 134: 979-994.

Xu H, Gao Q and Qi H (2009). Study of tissue culture and genetic transformation on blackcurrant. J. Fruit Sci. 26: 190-193.

Genetics and Molecular Research 14 (1): 2726-2734 (2015)

CFUNPEC-RP www.funpecrp.com.br 